J. DIFFERENTIAL GEOMETRY

79 (2008) 25-32

\title{
ALMOST COMPLEX 4-MANIFOLDS WITH VANISHING FIRST CHERN CLASS
}

\author{
Stefan BAUER
}

\begin{abstract}
An odd Seiberg-Witten invariant imposes bounds on the signature of a closed, almost complex 4-manifold with vanishing first Chern class. This applies in particular to symplectic 4-manifolds of Kodaira dimension zero.
\end{abstract}

\section{Introduction}

Vanishing of the first Chern class imposes severe restrictions on a compact complex surface: It has to be minimal and of Kodaira dimension at most zero. The list of examples [1], p. 188, is rather short and known to be complete $([\mathbf{1 0}],[\mathbf{1 5}])$. It comprises in particular $K 3$ surfaces and tori, but also other examples found by and named after Bombieri, Inoue, Hopf and Kodaira. Amongst these surfaces, only the $K 3$-surfaces exhibit nonvanishing signature.

Including closed symplectic 4-manifolds into the consideration, a few more examples of such with vanishing first Chern class become available $[\mathbf{1 6}],[\mathbf{7}],[\mathbf{8}]$. However, $K 3$-surfaces remain the only known examples with nonvanishing signature. The main result of this paper relates this more or less empirical fact to Seiberg-Witten theory.

Theorem 1.1. Let $X$ be a closed, almost complex 4-manifold with vanishing first Chern class. If the dimension $b_{2}^{+}(X)$ of a maximal positive definite linear subspace in the second cohomology of $X$ satisfies $b_{2}^{+}(X) \geq 4$, then the Seiberg-Witten invariant of $X$ is an even number.

According to a theorem of Taubes [14], the absolute value of the Seiberg-Witten invariant of a symplectic 4-manifold is 1 , as soon as $b_{2}^{+}(X) \geq 2$. So this theorem applies, in particular, to compact symplectic 4-manifolds.

Corollary 1.2. A closed, symplectic 4-manifold $X$ with torsion first Chern class satisfies the inequality

$$
b_{2}^{+}(X) \leq 3
$$

Received 03/29/2006. 
Indeed, if the first Chern class of $X$ is torsion, then there is a finite covering $\tilde{X}$ with vanishing first Chern class, which of course is symplectic. The induced map $H^{2}(X ; \mathbb{R}) \rightarrow H^{2}(\tilde{X} ; \mathbb{R})$ is injective.

Remark 1.3. Let $X$ be an almost complex manifold with vanishing first Chern class and $b_{2}^{+}(X) \leq 3$. Then the signature is either zero or -16 . If the signature is -16 , then $b_{2}^{+}(X)=3$ and the first Betti number vanishes. Otherwise the Betti numbers are related through $b_{1}=1+b_{2}^{+}$.

Indeed, according to Rochlin's theorem the signature is divisible by 16. The assumption thus implies an upper bound 0 for the signature of $X$. In the equality $c_{1}^{2}-2 c_{2}=p_{1}$ of characteristic classes, the second Chern class equals the Euler class of an almost complex manifold and the first Pontrjagin class describes three times the signature. So we obtain an equality

$$
\begin{aligned}
0=\left\langle 2 c_{2}+p_{1},[X]\right\rangle & =2\left(2-2 b_{1}+b_{2}^{+}+b_{2}^{-}\right)+3\left(b_{2}^{+}-b_{2}^{-}\right) \\
& =4\left(1-b_{1}+b_{2}^{+}\right)+\operatorname{sign}(X),
\end{aligned}
$$

from which we conclude $\operatorname{sign}(\mathrm{X}) \geq-16$ and the claimed values of the Betti numbers.

Corollary 1.4. Let $X$ be a closed symplectic 4-manifold with vanishing first Chern class and $\operatorname{sign}(\mathrm{X})=-16$. Then the fundamental group of $X$ has no proper subgroup of finite index.

Indeed, the covering manifold $\tilde{X}$ associated to a subgroup of finite index $n$ would be compact symplectic with $c_{1}(\tilde{X})=0$ and with signature $\operatorname{sign}(\tilde{X})=n \cdot \operatorname{sign}(X)$.

Fundamental groups of complex surfaces are rather restricted. In contrast, every finitely presented group can be realized as the fundamental group of a symplectic 4-manifold [9]. But, doesn't any finitely generated group contain subgroups of finite index? The amazing answer is: No. In 1965, Richard Thompson constructed infinite simple groups which are finitely presented; compare [5]. If such a Thompson group $T$ did admit a proper subgroup $S$ of finite index, then the kernel of the action of $T$ on $T / S$ would be of finite index, contradicting simplicity.

Question 1.5. Is there a symplectic homology- $K 3$-surface with vanishing first Chern class and nontrivial fundamental group? More specifically, can a Thompson group be the fundamental group of such a manifold?

The fundamental group of a symplectic manifold with vanishing first Chern class and vanishing signature has a property corresponding to 1.4: Any subgroup of finite index has rank at most 4. Of course, this narrows the range of possible fundamental groups of such manifolds. But still there is a considerable gap if one compares with the groups 
known to be realizable by symplectic manifolds of Kodaira dimension zero.

Partial results with regard to 1.2 were obtained by Morgan-Szabo [12] under the assumption $b_{1}(X)=0$, and by Tian-Jun Li [11] under the assumption $b_{1}(X) \leq 4$ (compare also [13]). The main theorem above partially answers Question 9.7 in $[\mathbf{3}]$ and proves the "Betti Number Conjecture" in $[\mathbf{1 1}]$.

The proof of the main result in the present paper is modelled on the stable cohomotopy proof [3], Thm. 9.5, of Morgan-Szabo's result. The concept can be explained in a few words: In its stable homotopy interpretation $([\mathbf{2}],[\mathbf{4}],[\mathbf{3}])$, the Seiberg-Witten invariant is the degree of a monopole map. Source and target depend on index data of the given 4-manifold in a controllable way. So it suffices to show that under the assumptions of the theorem, there are only maps of even degree between the relevant spaces. This follows from equivariant obstruction theory using the fact that the vanishing of the first Chern class leads to additional symmetry of the monopole map.

Acknowledgement. I am grateful to Katrin Tent for helpful remarks on group theory.

\section{Proof of the main theorem}

Due to the vanishing of the integral first Chern class, the almost complex 4-manifold $X$ may be equipped with a spin structure. Fixing such a spin structure and, furthermore, a Riemannian metric on $X$, gives rise to a monopole map and a refined Seiberg-Witten invariant. A key observation, well known in gauge theory, is that for spin-manifolds the monopole map is actually $\operatorname{Pin}(2)$-equivariant, where $\operatorname{Pin}(2) \subset S p(1) \subset$ $\mathbb{H}^{*}$ is the normalizer of the maximal torus $\mathbb{T}=S p(1) \cap \mathbb{C}^{*}$ in the group $S p(1)$ of quaternions of unit length. As explained in [3, Ch. 9], the refined invariant $[\mu]$ is a morphism in the Pin (2)-Spanier-Whitehead category indexed by a universe $\mathcal{U}$ containing only the quaternions $\mathbb{H}$ and the real 1-dimensional nontrivial Pin (2)-representation $V$ as irreducible summands. The monopole morphism

$$
[\mu] \in\left\{T(\operatorname{ind} D), S^{H^{+}(X)}\right\}_{\mathcal{U}}^{\operatorname{Pin}(2)}
$$

is a morphism from a Thom spectrum associated to the virtual index bundle of a family of Dirac operators to a sphere spectrum. Source and target will be detailed in an instant.

Twisting the Dirac operator associated to the fixed spin structure with flat $\mathbb{T}$-connections defines a family of Dirac operators, parametrized by the torus

$$
\operatorname{Pic}^{0}(X)=H^{1}(X ; \mathbb{R}) / H^{1}(X ; \mathbb{Z}) .
$$

The untwisted Dirac operator itself is $S p(1)$-equivariant. This symmetry reduces to a Pin (2)-symmetry over the given parameter space, with 
$j \in \operatorname{Pin}(2)$ acting on $\operatorname{Pic}^{0}(X)$ via multiplication by -1 . So the virtual index bundle can be represented as a difference

$$
\operatorname{ind} D=F_{0}-F_{1}
$$

of complex vector bundles with quaternionic structures (i.e., equipped with complex anti-linear bundle maps $j$ with $j^{2}=-1$ over the given involution of the base) and thus an element in the Grothendieck group $K Q\left(\operatorname{Pic}^{0}(X)\right)$ of such bundles. Without loss of generality one can assume $F_{1}$ to be trivial $F_{1}=\underline{\mathbb{H}}^{c}:=\operatorname{Pic}^{0}(X) \times \mathbb{H}^{c}$. The rank of $F_{0}$ as a complex bundle is determined by index theory:

$$
\mathrm{r} k_{\mathbb{C}} F_{0}=\frac{-\operatorname{sign}(\mathrm{X})}{8}+2 c .
$$

The target of the monopole morphism is a sphere spectrum $S^{H^{+}(X)}$, the suspension spectrum of the one-point completed $b_{2}^{+}(X)=b$-dimensional vector space $H^{+}(X)$ of self-dual harmonic 2-forms on $X$. The $\operatorname{Pin}(2)$-action factors through the quotient $\mathbb{Z} / 2$ with $j$ acting by multiplication with -1 ; so we may identify $H^{+}(X) \cong V^{b}$.

In particular, the monopole morphism is represented by a Pin (2)equivariant based map

$$
\mu: T\left(F_{0}\right) \wedge S^{V^{d}} \rightarrow S^{\mathbb{H}^{c}+V^{b+d}} .
$$

We are going to classify the relevant Pin(2)-equivariant maps from Thom spaces to spheres as above using equivariant obstruction theory as in [6], ch. II.3. For this we need a slightly more general setup:

\section{Notation 2.1.}

1) Let $F$ be a complex vector bundle with quaternionic structure over $\operatorname{Pic}^{0}(X)$ and let $U \hookrightarrow W$ be a fixed linear inclusion of Pin (2)representations $U \cong V^{d_{0}}$ and $W \cong \mathbb{H}^{c}+V^{d_{1}}$. We will call the pair $\left(T \tilde{F}, S^{W}\right)$ consisting of the Thom space of the bundle $\tilde{F}=F+\underline{U}$ and the 1-point compactification $S^{W}$ of $W$ an Euler pair of index $(\xi, n)$ with $\xi=F-\underline{\mathbb{H}}^{c} \in K Q\left(\operatorname{Pic}^{0}(X)\right)$ and $n=d_{1}-d_{0} \geq 0$. The virtual dimension of the Euler pair is the difference $\operatorname{dim}(T \tilde{F})-$ $\operatorname{dim}\left(S^{W}\right)=b_{1}(X)+2 \operatorname{rk}_{\mathbb{C}}(\xi)-\mathrm{n}$ of the dimensions of the two spaces.

2) A map $f: T \tilde{F} \rightarrow S^{W}$ for an Euler pair $\left(T \tilde{F}, S^{W}\right)$ will be called a quasipole map, if its restriction to the $\mathbb{T}$-fixed point set is the 1-point completion of the projection to the fiber

$$
\operatorname{Pic}^{0}(X) \times U \rightarrow U \hookrightarrow W^{\mathbb{T}} .
$$

A quasipole map need not be Pin(2)-equivariant. The set of homotopy classes of $G$-equivariant quasipole maps for subgroups $G \leq \operatorname{Pin}(2)$ will be denoted by $\left[T \tilde{F}, S^{W}\right]_{q}^{G}$.

The following lemma lists a few easy observations: 
Lemma 2.2. Let $\left(T \tilde{F}, S^{W}\right)$ be an Euler pair.

1) The space $T \tilde{F}$ can be equipped with the structure of a Pin(2)equivariant $C W$-complex. The space $S^{W}$ is a sphere with a linear Pin (2)-action.

2) The $\mathbb{T}$-fixed point set $T \tilde{F}^{\mathbb{T}} \subset T \tilde{F}$ is the Thom space $T \underline{U}$ of the trivial bundle $\underline{U}=\operatorname{Pic}^{0}(X) \times U$. The residual $\mathbb{Z} / 2=\operatorname{Pin}(2) / \mathbb{T}$ action is via multiplication by -1 on both base and fiber of $\underline{U}$.

3) If the virtual dimension of $\left(T \tilde{F}, S^{W}\right)$ is 1 and $\operatorname{dim}(W)=w$, then the cohomology group $H^{w}(T \tilde{F} / \mathbb{T}, T \underline{U} ; \mathbb{Z})$ is isomorphic to $\mathbb{Z}$.

4) Let $X$ be an almost complex manifold with vanishing first Chern class. Then the monopole morphism is represented by a Pin (2)equivariant quasipole map on an Euler pair of index (ind $\left.D, b_{2}^{+}(X)\right)$ and virtual dimension 1 .

Proof. After introducing an equivariant metric on the bundle $F+\underline{U}$, the unit disc bundle is a manifold with a differentiable Pin (2)-action and thus can be given an equivariant $\mathrm{CW}$-structure such that the sphere bundle is a sub-complex. Such a CW-structure induces one on the Thom space.

If an Euler pair is of virtual dimension 1, then after replacing $T \underline{U}$ by a tubular neighborhood, $H^{*}(T \tilde{F} / \mathbb{T}, T \underline{U} ; \mathbb{Z})$ is the cohomology of a connected and orientable manifold of dimension $w$ relative to its boundary.

The monopole morphism for a 4-manifold is linear when restricted to the $\mathbb{T}$-fixed point sets and satisfies the defining condition of a quasipole map, cf. [2], [3]. For an almost complex manifold, the virtual dimension of an Euler pair with index $\left(\operatorname{ind} D, b_{2}^{+}(X)\right)$ is (compare 1.2)

$$
b_{1}(X)-\frac{\operatorname{sign}(\mathrm{X})}{4}-b_{2}^{+}(X)=1 \text {. }
$$

q.e.d.

In $[\mathbf{3}]$ ch.4, a degree homomorphism

$$
h:\left[T \tilde{F}, S^{W}\right]_{q}^{\mathbb{T}} \rightarrow \mathbb{Z}
$$

was defined for an Euler pair of index $(\xi, n)$ with $n \geq 2$, the sign depending on a choice of orientations. The Seiberg-Witten invariant of $X$ is the degree of the monopole morphism. The degree in the case of virtual dimension 1 is defined as follows: The condition $n \geq 2$ implies a natural one-to-one correspondence

$$
\left[T \tilde{F}, S^{W}\right]_{q}^{\mathbb{T}} \cong\left[T \tilde{F} / T \underline{U}, S^{W}\right]^{\mathbb{T}}
$$

of $\mathbb{T}$-homotopy classes of quasipole maps with $\mathbb{T}$-homotopy classes of maps which are constant on the $\mathbb{T}$-fixed point set. An element $f$ of the latter set induces a homomorphism in reduced $\mathbb{T}$-equivariant Borel 
cohomology

$$
f^{*}: \tilde{H}_{\mathbb{T}}^{*}\left(S^{W} ; \mathbb{Z}\right) \rightarrow H_{\mathbb{T}}^{*}(T \tilde{F}, T \underline{U} ; \mathbb{Z})=H^{*}(T \tilde{F} / \mathbb{T}, T \underline{U} ; \mathbb{Z}) .
$$

The image $f^{*}([W]) \in H^{w}(T \tilde{F} / \mathbb{T}, T \underline{U} ; \mathbb{Z}) \cong \mathbb{Z}$ of the generator $[W] \in$ $\tilde{H}_{\mathbb{T}}^{w}\left(S^{W} ; \mathbb{Z}\right)$ of $\tilde{H}_{\mathbb{T}}^{*}\left(S^{W} ; \mathbb{Z}\right)$ as a free $H_{\mathbb{T}}^{*}(p t ; \mathbb{Z})$-module is the degree of $f$.

Lemma 2.3. Let $\left(T \tilde{F}, S^{W}\right)$ be an Euler pair of index $(\xi, n)$ and virtual dimension 1 .

1) There exists a Pin(2)-equivariant quasipole map $f: T \tilde{F} \rightarrow S^{W}$.

2) If $n \geq 2$, then the degree map $h$ induces a bijection

$$
\left[T \tilde{F}, S^{W}\right]_{q}^{\mathbb{T}} \cong \mathbb{Z}
$$

3) Suppose $n \geq 2$ and both $f$ and $g$ are Pin(2)-equivariant quasipole maps on the given Euler pair. Then the degrees of $f$ and $g$ differ by an even number.

Proof. We have to show that the given map on the $\mathbb{T}$-fixed points extends to a Pin (2)-equivariant map over $T \tilde{F}$. The obstructions to extending over the $l$-skeleton of $T \tilde{F}$ are elements of obstruction groups

$$
\mathfrak{H}_{\operatorname{Pin}(2)}^{k}\left(T \tilde{F}, T \underline{U} ; \pi_{k-1}\left(S^{W}\right)\right)
$$

(as defined in [6], II.3) for $k \leq l$. As long as $l \leq w$ holds, these obstruction groups are zero due to the vanishing of the coefficient groups. But the $w$-skeleton of $T \tilde{F}$ already is the whole of $T \tilde{F}$ (a free Pin (2)equivariant $k$-cell $\operatorname{Pin}(2) \times D^{k}$ has topological dimension $\left.k+1\right)$. This proves the first part of the lemma.

If $n \geq 2$, then we may use the natural one-to-one correspondence

$$
\left[T \tilde{F}, S^{W}\right]_{q}^{\mathbb{T}} \cong\left[T \tilde{F} / T \underline{U}, S^{W}\right]^{\mathbb{T}}
$$

to single out a nullhomotopic $\mathbb{T}$-equivariant quasipole map. Associating to a $\mathbb{T}$-equivariant quasipole map $f$ the difference cocycle to the nullhomotopic quasipole map defines (thm II.3.17 in [6]) a bijective map

$$
\left[T \tilde{F}, S^{W}\right]_{q}^{\mathbb{T}} \rightarrow \mathfrak{H}_{\mathbb{T}}^{w}\left(T \tilde{F}, T \underline{U} ; \pi_{w}\left(S^{W}\right)\right) \cong H^{w}(T \tilde{F} / \mathbb{T}, T \underline{U} ; \mathbb{Z}) .
$$

Composing an isomorphism of the latter group with $\mathbb{Z}$ results in the degree map $h$. This proves the second part of the lemma.

Obstruction theory associates to the Pin (2)-equivariant quasipole maps $f$ and $g$ a difference cocycle and thus an element in the group $\mathfrak{H}_{\operatorname{Pin}(2)}^{w}\left(T \tilde{F}, T \underline{U} ; \pi_{w}\left(S^{W}\right)\right)$. The image of the restriction homomorphism

$$
\mathfrak{H}_{\operatorname{Pin}(2)}^{w}\left(T \tilde{F}, T \underline{U} ; \pi_{w}\left(S^{W}\right)\right) \rightarrow \mathfrak{H}_{\mathbb{T}}^{w}\left(T \tilde{F}, T \underline{U} ; \pi_{w}\left(S^{W}\right)\right) \cong \mathbb{Z}
$$

consists of the multiples of $2=|\operatorname{Pin}(2) / \mathbb{T}|$ by $[6]$, Prop. 4.9. $\quad$ q.e.d.

The proof of the theorem uses the Pin (2)-equivariant Hopf map $\eta$ : $S^{\mathbb{H}} \rightarrow S^{V^{3}}$. Suppose the action of Pin (2) on $\mathbb{H}$ is by left multiplication, 
and consider $V^{3} \subset \mathbb{H}$ embedded as purely imaginary quaternions. Then the Hopf map is given by $\eta(h)=\bar{h} i h$.

We are now ready to finish the proof of the main theorem:

Proof. Let $X$ be an almost complex manifold with vanishing first Chern class and $b=b_{2}^{+}(X) \geq 4$. We will show that the degree of every the Pin (2)-equivariant quasipole map and thus the degree of the monopole morphism is even. Because of the last part of 2.3 , it suffices to exhibit a Pin (2)-equivariant quasipole map of even degree on an Euler pair of index $(\operatorname{ind} D, b)$. For this we choose an Euler pair $\left(T \tilde{F}, S^{W}\right)$ of index (ind $D-\underline{\mathbb{H}}, b-4$ ). According to the first part of 2.3 , there exists a Pin (2)-equivariant quasipole map $f: T \tilde{F} \rightarrow S^{W}$. The map $f \wedge \eta$ then is a Pin (2)-equivariant quasipole map $T(\tilde{F}+\underline{\mathbb{H}}) \rightarrow S^{W+V^{3}}$. Composed with the inclusion $S^{W+V^{3}} \hookrightarrow S^{W+V^{4}}$ we get a Pin (2)-equivariant

quasipole map of degree zero on the Euler pair $\left(T(\tilde{F}+\underline{\mathbb{H}}), S^{W+V^{4}}\right)$ of index $(\operatorname{ind} D, b)$.

q.e.d.

\section{References}

[1] W. Barth, C. Peters, \& A. van de Ven, Compact complex surfaces, Ergebnisse der Mathematik, Springer-Verlag, Berlin, 1984, MR 0749574, Zbl 1036.14016.

[2] S. Bauer, On connected sums of 4-manifolds, preprint, available at www. mathematik.uni-bielefeld/sfb343/preprints.

[3] _ Refined Seiberg-Witten invariants, in 'Different Faces of Geometry' (S.K. Donaldson, Y. Eliashberg, M. Gromov, eds.), Internat. Math. Ser., Kluwer Academic/Plenum Publishers, New York, 2004, MR 2102993, Zbl 1083.57039.

[4] S. Bauer \& M. Furuta, A stable cohomotopy refinement of Seiberg-Witten invariants, I, Invent. Math. 155 (2004) 1-19, MR 2025298, Zbl 1050.57024.

[5] J.W. Cannon, W.J. Floyd, \& W.R. Parry, Introductory notes on Richard Thompson's groups, Enseign. Math. (2) 42 (1996) 215-256, MR 1426438, Zbl 0880.20027.

[6] T. tom Dieck, Transformation Groups, de Gruyter, Berlin, 1987, MR 0889050, Zbl 0611.57002 .

[7] M. Fernández, M. Gotay, \& A. Gray, Compact parallelizable 4-dimensional symplectic and complex manifolds, Proc. Amer. Math.Soc. 103 (1988) 1209-1212, MR 0955011, Zbl 0656.53034.

[8] H.-J. Geiges, Symplectic structures on $T^{2}$-bundles over $T^{2}$, Duke Math. J. 67 (1992) 539-555, MR 1181312, Zbl 0763.53037.

[9] R.E. Gompf, A new construction of symplectic manifolds, Ann. of Math. (2) 142 (1995) 527-595, MR 1356781, Zbl 0849.53027.

[10] J. Li, S. Yau, \& F. Zheng, On projectively flat Hermitian manifolds, Comm. in Analysis and Geometry 2 (1994) 103-109, MR 1312680, Zbl 0837.53053.

[11] T.J. Li, Symplectic 4-manifolds with Kodaira dimension zero, J. Differential Geom. 74(2) (2006) 321-352, MR 2259057, Zbl 1105.57028. 
[12] J. Morgan \& Z. Szabo, Homotopy K3-surfaces and mod 2 Seiberg-Witten invariants, Math. Res. Lett. 4 (1997) 17-21, MR 1432806, Zbl 0876.57031.

[13] D. Ruberman \& S. Strle, Mod 2 Seiberg-Witten invariants fo homology tori, Math. Res. Lett. 7 (2000) 789-799, MR 1809302, Zbl 1005.57016.

[14] C.H. Taubes, The Seiberg-Witten invariants and symplectic forms, Math. Res. Letters 1 (1994) 809-822, MR 1306023, Zbl 0853.57019.

[15] A. Teleman, Projectively flat surfaces and Bogomolov's theorem on class $\mathrm{VII}_{\circ}-$ surfaces, Int. J. Math 5(2) (1994) 253-264, MR 1266285, Zbl 0803.53038.

[16] W. Thurston, Some simple examples of symplectic manifolds, Proc. Amer. Math. Soc. 55 (1976) 467-468, MR 0402764, Zbl 0324.53031.

[17] E. Witten, Monopoles and four-manifolds, Math. Res. Lett. 1 (1994) 769-796, MR 1306021, Zbl 0867.57029.

Note added in proof: Four months after submission of the present paper to JDG, I got notice of a preprint being circulated in which T.J. Li gives a proof of 1.1 and of 1.2. The preprint was published within two months. Its reference is:

[18] T.J. Li, Quaternionic bundles and Betti numbers of symplectic 4-manifolds with Kodaira dimension zero, IMRN, Vol. 2006, Article ID 37385, 1-28, MR 2264722.

FAKULTät FÜR MATHEMATIK UNIVERsität Bielefeld, PF 100131

D-33501 BIELEFELD

GERMANY

E-mail address: bauer@mathematik.uni-bielefeld.de 